\title{
Anomalous diffusion induced by a Mittag-Leffler correlated noise
}

\author{
A. D. Viñales ${ }^{1, *}$ and M. A. Despósito ${ }^{1,2}$ \\ ${ }^{1}$ Departamento de Física, Facultad de Ciencias Exactas y Naturales, Universidad de Buenos Aires, 1428 Buenos Aires, Argentina \\ ${ }^{2}$ Consejo Nacional de Investigaciones Científicas y Técnicas, CONICET, Buenos Aires, Argentina
}

(Received 21 October 2006; published 11 April 2007)

\begin{abstract}
We introduce a Mittag-Leffler correlated random force leading to anomalous diffusion. Starting from a generalized Langevin equation, and using Laplace analysis we derive exact expressions for the mean values, variances and diffusion coefficient for a free particle in terms of generalized Mittag-Leffler functions and its derivatives. The asymptotic behavior of these quantities are obtained, from which the anomalous diffusion behavior of the particle is displayed.
\end{abstract}

DOI: 10.1103/PhysRevE.75.042102

PACS number(s): 02.50.-r, 05.40.-a, 02.50.Ey

Anomalous diffusion, which has been observed in disordered media and other complex systems, has been the subject of numerous investigations in the last years, both experimental and theoretical [1]. In one dimension and in the absence of an external driving force, anomalous diffusion is characterized by the occurrence of a mean square displacement of the form $\left\langle X^{2}(t)\right\rangle \sim t^{\xi}$, which deviates from the linear Brownian dependence on time. According to the value of the anomalous diffusion exponent $\xi$, one distinguishes slow or subdiffusion $(0<\xi<1)$, and enhanced or superdiffusion $(\xi$ $>1$ ). The modeling of such anomalous diffusing stochastic processes has mainly been done within the framework of the fractional kinetic equation approach $[1,2]$ and the generalized Langevin equation (GLE) approach [3-6]. This last approach has been used recently in the description of diverse anomalous diffusion phenomena, such as conformational fluctuations within a single protein molecule $[7,8]$, reaction kinetics of single enzymes [9], and nuclear fusion reactions [10]. The GLE is a nonlocal equation that, in the absence of a deterministic field, can be written in the form

$$
\ddot{X}(t)+\int_{0}^{t} d t^{\prime} \gamma\left(t-t^{\prime}\right) \dot{X}\left(t^{\prime}\right)=F(t),
$$

where $X(t)$ represents the position of a particle of mass $m$ $=1$ at time $t, \gamma(t)$ is the dissipative memory kernel, and $F(t)$ is a zero-centered Gaussian and stationary random force. The correlation function of the random force obeys the fluctuation-dissipation theorem

$$
\left\langle F(t) F\left(t^{\prime}\right)\right\rangle=C\left(\left|t-t^{\prime}\right|\right)=k_{B} T \gamma\left(\left|t-t^{\prime}\right|\right),
$$

where $k_{B}$ is the Boltzmann constant, and $T$ is the absolute temperature of the environment.

The solution of the GLE (1) can be obtained by means of the Laplace transformation technique $[3,4]$,

$$
X(t)=\langle X(t)\rangle+\int_{0}^{t} d t^{\prime} G\left(t-t^{\prime}\right) F\left(t^{\prime}\right),
$$

\footnotetext{
*Electronic address: dvinales@df.uba.ar
}

$$
\dot{X}(t)=\langle\dot{X}(t)\rangle+\int_{0}^{t} d t^{\prime} g\left(t-t^{\prime}\right) F\left(t^{\prime}\right),
$$

where

$$
\begin{gathered}
\langle X(t)\rangle=x_{0}+v_{0} G(t), \\
\langle\dot{X}(t)\rangle=v_{0} g(t),
\end{gathered}
$$

being $x_{0}=X(t=0)$ and $v_{0}=\dot{X}(t=0)$ the (deterministic) initial position and velocity of the particle. The relaxation function $G(t)$ is the inverse form of the Laplace transform

$$
\hat{G}(s)=\frac{1}{s^{2}+\hat{\gamma}(s) s},
$$

where $\hat{\gamma}(s)$ is the Laplace transform of the dissipative memory kernel. The relaxation function $g(t)$ is the derivative of $G(t)$, i.e., $g(t)=G^{\prime}(t)$. Hence

$$
\hat{g}(s)=\frac{1}{s+\hat{\gamma}(s)}
$$

and from Eqs. (3) and (4) it follows that $G(0)=0$ and $g(0)$ $=1$.

Explicit expressions of the variances are given by [4]

$$
\begin{gathered}
\sigma_{x x}(t)=k_{B} T\left[2 I(t)-G^{2}(t)\right], \\
\sigma_{v v}(t)=k_{B} T\left[1-g^{2}(t)\right], \\
\sigma_{x v}(t)=k_{B} T G(t)[1-g(t)],
\end{gathered}
$$

where

$$
I(t)=\int_{0}^{t} d t^{\prime} G\left(t^{\prime}\right) .
$$

Alternatively, the second moments read as

$$
\left\langle X^{2}(t)\right\rangle=x_{0}^{2}+\left(v_{0}^{2}-k_{B} T\right) G^{2}(t)+2 k_{B} T I(t)+2 x_{0} v_{0} G(t),
$$

$$
\left\langle\dot{X}^{2}(t)\right\rangle=k_{B} T+\left(v_{0}^{2}-k_{B} T\right) g^{2}(t) .
$$

It is well known that if the correlation function (2) is a 
Dirac delta function, the stochastic process is Markovian and its dynamics can be straightforwardly obtained [11]. However, in order to describe the non-Markovian dynamics of an anomalously diffusing particle one must take into account the memory effects through a long-time tail noise. In particular, a power-law correlation function is usually employed to model the anomalous diffusion processes $[5,6,12-14]$. In this paper, we propose a more general correlation function modeled as

$$
C(t)=C_{0}(\lambda)(\tau)^{-\lambda} E_{\lambda}\left[-(|t| / \tau)^{\lambda}\right],
$$

where $\tau$ acts as a characteristic memory time and $C_{0}(\lambda)$ is a proportionality coefficient dependent on the exponent $\lambda$ but independent of time. The exponent $\lambda$ can be taken as $0<\lambda$ $<2$, which is determined by the dynamical mechanism of the physical process considered. The $E_{\alpha}(y)$ function denotes the Mittag-Leffler function [15] defined through the series

$$
E_{\alpha}(y)=\sum_{j=0}^{\infty} \frac{y^{j}}{\Gamma(\alpha j+1)}, \quad \alpha>0 .
$$

In what follows we only deal with times $t \geqslant 0$. Then, we omit the modulus in the argument of functions for simple notational convenience.

From the asymptotic behaviors of the Mittag-Leffler function [16] one easily can deduce that, for $\lambda \neq 1$, the correlation function (15) behaves as a stretched exponential for short times and as an inverse power law in the long time regime. It is worth pointing out that the function $E_{\lambda}\left(-t^{\lambda}\right)$ exhibits different behaviors depending on the value of $\lambda$ [16]. If $0<\lambda$ $<1, E_{\lambda}\left(-t^{\lambda}\right)$ is a completely monotone function and tends to zero from above as $t$ tends to infinity. If $1<\lambda<2, E_{\lambda}\left(-t^{\lambda}\right)$ can be decomposed in a completely monotone function which tends to zero from below as $t$ tends to infinity plus an oscillatory contribution with an exponentially decreasing amplitude. On the other hand, setting $\lambda=1$ the correlation function (15) reduces to an exponential form which describes a standard Ornstein-Uhlenbeck process [11].

Note that in the limit $\tau \rightarrow 0$ the proposed correlation function (15) reproduce a power-law correlation function

$$
C(t)=C_{0}(\lambda) t^{-\lambda} / \Gamma(1-\lambda),
$$

which has been obtained introducing the asymptotic behavior at large $y$ of the Mittag-Leffler function [16]

$$
E_{\alpha}(-y) \sim[y \Gamma(1-\alpha)]^{-1}, \quad y>0
$$

in expression (15). Moreover, taking the limit $\lambda \rightarrow 1$ in Eq. (17) and using the formal representation of the generalized Dirac delta [17] we obtain

$$
C(t)=C_{0}(1) \delta(t),
$$

which corresponds to a white noise, nonretarded friction, and standard Brownian motion [11].

Now we obtain analytical expressions for the kernels $I(t)$, $G(t)$, and $g(t)$, for $\lambda \neq 1$ assuming that the autocorrelation function is of the form (15). From (2), the memory kernel $\gamma(t)$ can be written as

$$
\gamma(t)=\gamma_{\lambda} E_{\lambda}\left[-(t / \tau)^{\lambda}\right] / \tau^{\lambda},
$$

where $\gamma_{\lambda}=C_{0}(\lambda) / k_{B} T$.

Then, its Laplace transform reads [16]

$$
\hat{\gamma}(s)=\frac{\gamma_{\lambda} s^{\lambda-1}}{1+s^{\lambda} \tau^{\lambda}} .
$$

Thus the kernel integral $I(t)$ is the Laplace inversion of

$$
\hat{I}(s)=\frac{\hat{G}(s)}{s}=\hat{I}_{0}(s)+\hat{I}_{1}(s),
$$

where

$$
\begin{gathered}
\hat{I_{0}}(s)=\frac{s^{-1} \tau^{\lambda}}{\tau^{\lambda} s^{2}+s^{2-\lambda}+\gamma_{\lambda}}, \\
\hat{I}_{1}(s)=\tau^{-\lambda} s^{-\lambda} \hat{I}_{0}(s) .
\end{gathered}
$$

Using the recipes given in Ref. [18] we get

$$
\begin{gathered}
I_{0}(t)=\sum_{k=0}^{\infty} \frac{(-1)^{k}}{k !}\left(\frac{\gamma_{\lambda}}{\tau^{\lambda}}\right)^{k} t^{2(k+1)} E_{\lambda, 3+(2-\lambda) k}^{(k)}\left[-(t / \tau)^{\lambda}\right], \\
I_{1}(t)=(t / \tau)^{\lambda} \sum_{k=0}^{\infty} \frac{(-1)^{k}}{k !}\left(\frac{\gamma_{\lambda}}{\tau^{\lambda}}\right)^{k} t^{2(k+1)} E_{\lambda, 3+\lambda+(2-\lambda) k}^{(k)}\left[-(t / \tau)^{\lambda}\right],
\end{gathered}
$$

where $E_{\alpha, \beta}(y)$ is the generalized Mittag-Leffler function [15] defined by the series expansion

$$
E_{\alpha, \beta}(y)=\sum_{j=0}^{\infty} \frac{y^{j}}{\Gamma(\alpha j+\beta)}, \quad \alpha>0, \quad \beta>0,
$$

and $E_{\alpha, \beta}^{(k)}(y)$ is the derivative of the Mittag-Leffler function

$$
E_{\alpha, \beta}^{(k)}(y)=\frac{d^{k}}{d y^{k}} E_{\alpha, \beta}(y)=\sum_{j=0}^{\infty} \frac{(j+k) ! y^{j}}{j ! \Gamma(\alpha(j+k)+\beta)} .
$$

The kernels $G(t)$ and $g(t)$ can be calculated using the relation [18]

$$
\frac{d}{d t}\left[t^{\alpha k+\beta-1} E_{\alpha, \beta}^{(k)}\left(-\gamma t^{\alpha}\right)\right]=t^{\alpha k+\beta-2} E_{\alpha, \beta-1}^{(k)}\left(-\gamma t^{\alpha}\right) .
$$

Then $G(t)=G_{0}(t)+G_{1}(t)$ where

$$
\begin{gathered}
G_{0}(t)=\sum_{k=0}^{\infty} \frac{(-1)^{k}}{k !}\left(\frac{\gamma_{\lambda}}{\tau^{\lambda}}\right)^{k} t^{2 k+1} E_{\lambda, 2+(2-\lambda) k}^{(k)}\left[-(t / \tau)^{\lambda}\right], \\
G_{1}(t)=(t / \tau)^{\lambda} \sum_{k=0}^{\infty} \frac{(-1)^{k}}{k !}\left(\frac{\gamma_{\lambda}}{\tau^{\lambda}}\right)^{k} t^{2 k+1} E_{\lambda, 2+\lambda+(2-\lambda) k}^{(k)}\left[-(t / \tau)^{\lambda}\right],
\end{gathered}
$$

and $g(t)=g_{0}(t)+g_{1}(t)$, where

$$
g_{0}(t)=\sum_{k=0}^{\infty} \frac{(-1)^{k}}{k !}\left(\frac{\gamma_{\lambda}}{\tau^{\lambda}}\right)^{k} t^{2 k} E_{\lambda, 1+(2-\lambda) k}^{(k)}\left[-(t / \tau)^{\lambda}\right],
$$




$$
g_{1}(t)=(t / \tau)^{\lambda} \sum_{k=0}^{\infty} \frac{(-1)^{k}}{k !}\left(\frac{\gamma_{\lambda}}{\tau^{\lambda}}\right)^{k} t^{2 k} E_{\lambda, 1+\lambda+(2-\lambda) k}^{(k)}\left[-(t / \tau)^{\lambda}\right] .
$$

The analytical expressions (25), (26), and (30)-(33) are the main result of this work. They fully determine the temporal evolution of the mean values of the position and the velocity as well as the variances of the process.

Now we analyze the behavior of the kernels $I(t), G(t)$, and $g(t)$ at times larger than the noise characteristic time $\tau$. This can be achieved introducing the asymptotic behaviors of the generalized Mittag-Leffler function [18]

$$
E_{\alpha, \beta}(-y) \sim \frac{1}{y \Gamma(\beta-\alpha)}, \quad y>0,
$$

and its derivative

$$
E_{\alpha, \beta}^{(k)}(-y) \sim(-1)^{k} \frac{k !}{y^{k+1}} \frac{1}{\Gamma(\beta-\alpha)}
$$

in Eqs. (25) and (26). After some algebra, we obtain for $t$ $\gg \tau$,

$$
I(t) \approx t^{2} E_{2-\lambda, 3}\left[-\left(\omega_{\lambda} t\right)^{2-\lambda}\right]+\frac{\tau^{\lambda}}{\gamma_{\lambda}}\left\{1-E_{2-\lambda}\left[-\left(\omega_{\lambda} t\right)^{2-\lambda}\right]\right\},
$$

where $\left(\omega_{\lambda}\right)^{2-\lambda}=\gamma_{\lambda}$. With the help of relation (29), the asymptotic expressions for $G(t)$ and $g(t)$ are given by

$$
\begin{aligned}
& G(t) \approx t E_{2-\lambda, 2}\left[-\left(\omega_{\lambda} t\right)^{2-\lambda}\right]-\frac{\tau^{\lambda}}{\gamma_{\lambda}} \frac{d}{d t} E_{2-\lambda}\left[-\left(\omega_{\lambda} t\right)^{2-\lambda}\right], \\
& g(t) \approx E_{2-\lambda}\left[-\left(\omega_{\lambda} t\right)^{2-\lambda}\right]-\frac{\tau^{\lambda}}{\gamma_{\lambda}} \frac{d^{2}}{d^{2} t} E_{2-\lambda}\left[-\left(\omega_{\lambda} t\right)^{2-\lambda}\right] .
\end{aligned}
$$

It is worth pointing out that the expansion used to obtain Eqs. (36)-(38) naturally introduces the characteristic time $\omega_{\lambda}^{-1}$. Note that the first three terms of the right-hand side of Eqs. (36)-(38), which come from $I_{1}(t), G_{1}(t)$, and $g_{1}(t)$, respectively, correspond to the exact expressions for the kernels obtained in the case of a pure power-law correlation function $[5,6,13,14,19]$. On the other hand, the other three terms come from the behavior of the correlation function at intermediate times $\tau<t<\omega_{\lambda}^{-1}$.

Now we consider the evolution of the second moment (14) in the intermediate time interval. Without loss of generality we suppose that $x_{0}=0$ and the thermal equilibrium condition $v_{0}^{2}=k_{B} T$. In this case, from Eq. (13) we obtain

$$
\left\langle X^{2}(t)\right\rangle=2 k_{B} T I(t) .
$$

Then, the diffusive behavior is completely established by the time behavior of the integral kernel $I(t)$. For times $t \gg \tau$ we obtain

$$
\begin{aligned}
\left\langle X^{2}(t)\right\rangle \approx & 2 k_{B} T t^{2} E_{2-\lambda, 3}\left[-\left(\omega_{\lambda} t\right)^{2-\lambda}\right] \\
& +2 k_{B} T \frac{\tau^{\lambda}}{\gamma_{\lambda}}\left\{1-E_{2-\lambda}\left[-\left(\omega_{\lambda} t\right)^{2-\lambda}\right]\right\} .
\end{aligned}
$$

In this situation, the time-dependent diffusion coefficient, defined by [5]

$$
D(t)=\frac{1}{2} \frac{d}{d t}\left\langle X^{2}(t)\right\rangle
$$

can be written as

$$
D(t) \approx k_{B} T t E_{2-\lambda, 2}\left[-\left(\omega_{\lambda} t\right)^{2-\lambda}\right]-k_{B} T \frac{\tau^{\lambda}}{\gamma_{\lambda}} \frac{d}{d t} E_{2-\lambda}\left[-\left(\omega_{\lambda} t\right)^{2-\lambda}\right] .
$$

It is worth pointing out that in this intermediate time interval $\left(\tau<t<\omega_{\lambda}^{-1}\right)$ the diffusion coefficient exhibits an additional term in relation to the expression given in Ref. [5] for a pure power-law correlation function.

In what follows we analyze the behavior of the kernels at times larger than the characteristic time $\omega_{\lambda}^{-1}$. Taking into account that $E_{\alpha}(y)=E_{\alpha, 1}(y)$, one can use again the asymptotic behavior of the generalized Mittag-Leffler function (34) for $\omega_{\lambda} t \gg 1$ to obtain

$$
\begin{gathered}
I(t) \approx \frac{1}{\gamma_{\lambda} \Gamma(\lambda+1)} t^{\lambda}, \\
G(t) \approx \frac{t^{\lambda-1}}{\gamma_{\lambda} \Gamma(\lambda)}, \\
g(t) \approx \frac{t^{(\lambda-2)}}{\gamma_{\lambda} \Gamma(\lambda-1)} .
\end{gathered}
$$

Introducing these asymptotic expansions into Eqs. (9)-(11) we can realize that these expressions correspond to the asymptotic variances obtained with the kernel (17), previously reported in Refs. $[5,13,14]$. In particular,

$$
\left\langle X^{2}(t)\right\rangle \approx k_{B} T \frac{2}{\gamma_{\lambda} \Gamma(\lambda+1)} t^{\lambda}
$$

and

$$
D(t) \approx k_{B} T \frac{1}{\gamma_{\lambda} \Gamma(\lambda)} t^{\lambda-1}
$$

showing that, for $\omega_{\lambda} t \gg 1$, the particle motion is subdiffusive for $0<\lambda<1$ and superdiffusive for $1<\lambda<2$. These results are in agreement with those obtained in Ref. [20], where it is shown that for every noise kernel that behaves as $t^{-\lambda}$ for long times, the diffusion coefficient behaves as $t^{\lambda-1}$.

Finally, we want to discuss some issue related to the microscopic origin of the proposed noise. To address this point one can suppose that the random force $F(t)$ has its origin in a thermal bath composed of harmonic oscillators. In this situation the dissipative memory kernel is usually described in the continuous limit by the reservoir spectral density $J(\omega)$ [21], 


$$
\gamma(t)=\frac{2}{\pi} \int_{0}^{\infty} \frac{J(\omega)}{\omega} \cos (\omega t) d \omega .
$$

Inserting the integral representation [22]

$$
E_{\lambda}\left(-x^{\lambda}\right)=\frac{2}{\pi} \sin (\lambda \pi / 2) \int_{0}^{\infty} \frac{\omega^{\lambda-1} \cos (x \omega)}{1+2 \omega^{\lambda} \cos (\lambda \pi / 2)+\omega^{2 \lambda}} d \omega,
$$

in the expression (20) it follows that

$$
J(\omega)=\omega^{\lambda} f_{c}(\omega \tau),
$$

where $f_{c}$ is the cutoff function

$$
f_{c}(x)=\gamma_{\lambda} \frac{\sin (\lambda \pi / 2)}{1+2 x^{\lambda} \cos (\lambda \pi / 2)+x^{2 \lambda}} .
$$

The function $f_{c}$ is a high-frequency cutoff function of typical width $\tau^{-1}$. Then, the proposed noise can be consider as a special case of non-Ohmic models [21].

In summary, we have proposed and realized a MittagLeffler correlated noise which can lead to anomalous diffusion. For certain values of the parameters that characterize this noise one can reproduce a power-law correlation function, an exponential one and a white noise. The dynamics of the particle can be explicitly obtained in analytical form and shows several different behaviors compared with previous results based on white and other colored noises.

This work was partially supported by the Agencia Nacional de Promoción Cientifíca y Tecnológica (Grant No. PICT8450), FONCYT, Argentina.
[1] For a review, see R. Metzler and J. Klafter, Phys. Rep. 339, 1 (2000).

[2] R. Metzler, E. Barkai, and J. Klafter, Phys. Rev. Lett. 82, 3563 (1999).

[3] K. G. Wang and M. Tokuyama, Physica A 265, 341 (1999).

[4] J. M. Porra, K. G. Wang, and J. Masoliver, Phys. Rev. E 53, 5872 (1996).

[5] N. Pottier, Physica A 317, 371 (2003).

[6] A. D. Viñales and M. A. Despósito, Phys. Rev. E 73, 016111 (2006).

[7] S. C. Kou and X. S. Xie, Phys. Rev. Lett. 93, 180603 (2004).

[8] W. Min, G. Luo, B. J. Cherayil, S. C. Kou, and X. S. Xie, Phys. Rev. Lett. 94, 198302 (2005).

[9] S. Chaudhury and B. J. Cherayil, J. Chem. Phys. 125, 024904 (2006).

[10] J. D. Bao and Y. Z. Zhuo, Phys. Rev. C 67, 064606 (2003).

[11] H. Risken, The Fokker-Plank Equation (Springer-Verlag, Ber- lin, 1989).

[12] K. G. Wang, Phys. Rev. A 45, 833 (1992).

[13] R. Kupferman, J. Stat. Phys. 114, 291 (2004).

[14] E. Lutz, Phys. Rev. E 64, 051106 (2001).

[15] A. Erdelyi et al., Higher Transcendental Functions (Krieger, Malabar, 1981), Vol. 3.

[16] F. Mainardi and R. Gorenflo, J. Comput. Appl. Math. 118, 283 (2000).

[17] F. Mainardi et al., Physica A 287, 468 (2000).

[18] I. Podlubny, Fractional Differential Equations (Academic, London, 1999).

[19] K. S. Fa, Phys. Rev. E 73, 061104 (2006).

[20] R. Morgado, F. A. Oliveira, G. G. Batrouni, and A. Hansen, Phys. Rev. Lett. 89, 100601 (2002).

[21] U. Weiss, Quantum Dissipative Systems (World Scientific, Singapore, 1993).

[22] M. N. Berberan-Santos, J. Math. Chem. 38, 629 (2005). 